\section{Border contrast as a function of retinal locus*}

\author{
DAVID E. ANDERSON $\dagger$ \\ University of Oregon Medical School, Portland, Oregon 97201
}

When the visibility of a small test spot was measured by direct brightness matching at various locations along the white edge of a black and white border, it was found that the white background was perceived as becoming progessively brighter as the border was approached. The effect was greater when a corner was neared. Moreover, greater effects, i.e., larger differences between the edge effect and base level (no border) were found at distances farther removed from the fovea ( $0 \mathrm{deg} 12 \mathrm{~min}$ to $3 \mathrm{deg} 52 \mathrm{~min}$ ).

Contrast effects induced by complex stimulus patterns with spatial variation of luminance were reported as early as 1865 by Ernest Mach (1865). Mach demonstrated that bright and dark bands could be seen in a visual pattern that did not include corresponding regions of higher or lower luminance, and that, in some cases, did not even include any abrupt discontinuities in the distribution of luminance. The Mach band phenomenon is probably a manifestation of the same kind of processes that are considered responsible for the more usual kind of contrast effects.

Noting that the relevance of adapting field size for contrast effects had been amply demonstrated (Blachowski, 1913; Ratoosh \& Graham, 1951; Steinhardt, 1936), Brown and Mueller (1965, pp. 219-220) concluded that it is not just the adaptation of the region stimulated by the test field that is important but that a considerable portion of the surrounding region also influences brightness discrimination and contrast effects.

Several recent experiments provide information concerning the effect of an abrupt boundary in an adapting field on increment thresholds. While investigating discrimination thresholds for small test spots as a function of their location in relation to sharp boundaries in the adapting field, Harms and Aulhorn (1955) and Fiorentini, Jeanne, and Toraldo di Francia (1955) found that in the region of a boundary between a light area and a darker area, a bright band may be perceived along the brighter side and a dark band may be perceived along the border on the dark side. Similar results were reported by Payne and Anderson (1969a, 1969b) for the bright side of the border.

There is some evidence to show that these border phenomena vary as a function

*This investigation was supported in part by funds provided for biological and medical research by the State of Washington Initiative Measure No. 171.

$\dagger$ Data reported here were collected as part of the research used for the author's PhD dissertation at Washington State University, Pullman, Washington (W. I1. Palyne, advisor). of the location of the test and inducing fields on the retina. Alpern (1953) found evidence to indicate that peripheral-inducing fields produce a greater contrast effect than do central-inducing fields. Aulhorn and Harms (1956) maintain that changes in sensitivity on the light side of a boundary are greater at locations further removed from the fovea. The effects of various retinal locations on border or edge phenomena have yet to be investigated systematically, however. The present study investigated elevations in increment thresholds, i.e., changes in subjective brightness of the background near borders, as a function of the retinal locus of the border. Measurements were confined to parafoveal regions of the retina.

\section{METHOD}

Apparatus

A movable $100 \%$-rag-vellum tracing-paper screen $79.3 \times 55.9 \mathrm{~cm}$ was strelched on a frame using a trampoline-type suspension. A Cartesian coordinate system of small dots with $1 \mathrm{~cm}$ separation was drawn on the screen. Black paper rectangles $(40.6 \times 30.5 \mathrm{~cm}-\mathrm{X}-\mathrm{Y}$ axis dimensions) were attached to the white viewing screen with small magnets. The test spot was projected on the back of the viewing screen through a tube with an inner diameter of $6 \mathrm{~mm}$ (visual angle of approximately $1 \mathrm{~min}$ ). The end of the tube was flush with the rear of the viewing screen and was covered with a $0.076 \mathrm{~mm}$ matte acetate film. Directly behind the viewing screen was a $128 \times 158 \mathrm{~cm}$ white field surrounded by blue drapes. The viewing screen was uniformly illuminated to a luminance of $4.4 \mathrm{~mL}$.

The test-spot illumination was furnished by a 420 -W current regulated quartz-halogen bulb (G. E. projection FAL V-line lamp) projected through a collimating lens, a neutral density optical wedge, a balancing wedge, and one or more neutral density filters before being projected on the back of the viewing screen. Test-spot luminance at the viewing screen (without any wedge or neutral density filter) was $952 \mathrm{~mL}$.
A Kodak Wratten No. 96 optical wedge (density range of 0.05 to 0.50 ) and appropriate balancing wedge were interposed between the light source and the viewing screen. The wedge was mounted on a sliding platform, which permitted the wedge to be moved from one side to the other in front of the light source, thus varying continuously the resultant intensity of the source. Up to six neutral density filters could be interposed in the light path to further attenuate the source. At least one filter ( 0.0 density) was used at all times.

A series of NE-2 neon fixation lights was placed in front of the viewing screen to the right of the test spot permitting stimulation in the parafoveal region of the upper retina with a range of $0 \mathrm{deg} 12 \mathrm{~min}$ to $3 \mathrm{deg} 52 \mathrm{~min}$ of visual angle. At a given retinal locus the test spot always remained at the same place; the background containing the black rectangles moved. Therefore no compensation for retinal sensitivity (at a given retinal locus) need be made.

The location of the optical wedge relative to the test spot (and, therefore, its density) was monitored using a 10-turn potentiometer attached to the drive shaft of the motor used to move the wedge. Three mercury batteries connected in series provided voltage bias on the potentiometer. This voltage was measured by a digital printing voltmeter that provided a paper record of the potentiometer's voltage readings.

\section{Observers}

The primary O (DA, corrected to normal vision) was a highly practiced $O$ with extensive experience at this type of task. A second, less practiced $O$ (DB, normal vision) was used to replicate portions of the results.

\section{Procedure}

The $O$ was seated in an ophthalmological chair equipped with a head rest $23.33 \mathrm{~m}$ from the viewing screen. The display was viewed binocularly through a 0.3 neutral density filter (Kodak Wratten No. 96) throughout all experimental conditions.

The test spot was flashed continuously (50-msec flash, $1 \mathrm{sec}$ between flashes). The $O$ 's task was to adjust the intensity of the flash until it matched that of the background. The match was accomplished by moving a switch, which, in turn, activated a motor moving the optical wedge. Neutral density filters were added (or removed) when the range of the wedge was insufficient. Five independent randomly-ordered observations were made at each combination of a Cartesian coordinate and a retinal locus. 


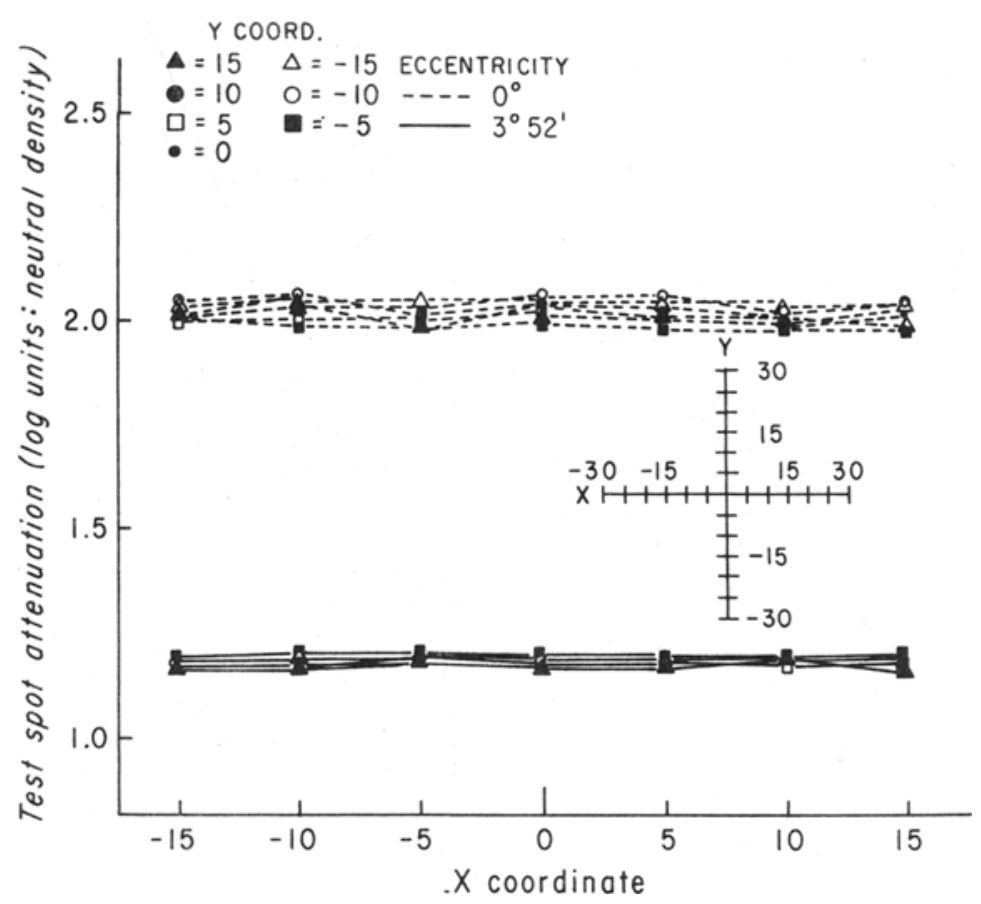

Fig. 1. Amount of attenuation (measured in density units) of the light source necessary to produce a brightness match between the test spot and the background as a function of the location of the test spot. Display consisted of no border (rectangle).

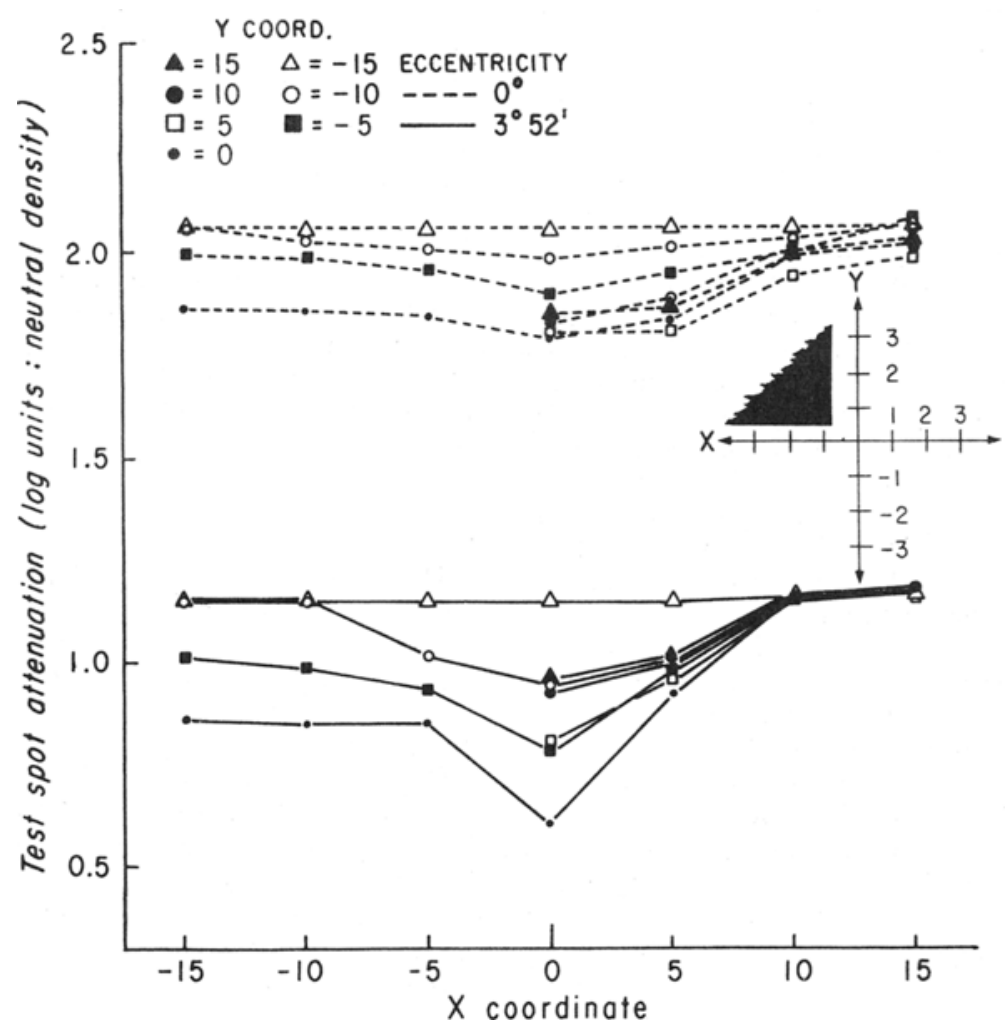

Fig. 2 Amount of attenuation (measured in density units) of the light source necessary to produce a brightness match between the test spot and the background as a function of the location of the test spot. Display consisted of one border (rectangle).

\section{Part One}

\section{RESULTS}

Changes in subjective background brightness as a function of test spot location for varying types of displays $(0,1$, 2 , and 3, rectangles) are given in Figs. 1-4. Subjective brightness of the background is given in terms of the amount of attenuation (measured in density units $-\mathrm{D}=\log 1 / \mathrm{T}$ where $T=$ transmittance of the filter) of the light source necessary to produce a brightness match between the test spot and the background. The larger the density unit, the dimmer the background appeared; the smaller the density unit, the brighter the background appeared. Each figure gives data for two retinal locations: $0 \mathrm{deg}$ $12 \mathrm{~min}$ and $3 \mathrm{deg} 52 \mathrm{~min}$.

Each data point on these (and subsequent figures) represent the median of five independent brightness matches by the primary 0 . Variation (range-highest value minus lowest value) among the five values ranged from 0.01 to 0.05 with the median variation being 0.02 . Data for a second $\mathrm{O}$ showed consistently higher values for all data points, but were of the same general shape and support all conclusions reached herein.

Investigating the test spot at various locations on the plain white screen ( 0 rectangles in the display) established subjective background brighteness when the retina was unaffected by a border in the field. These data are presented in Fig. 1. Data are shown for two retinal loci as a function of various locations of the test spot on the viewing screen. On the right-hand side of the figure a Cartesian coordinate system similar to that used in the actual display is shown. Figure 2 gives the results when a single black rectangle was placed in Quadrant 2 with the lower right-hand corner at $(-1,1)$. (See insert in Fig. 2.) The test spot must be made very bright to match the subjective background brightness along the lines $Y=0, Y=5$, and $Y=-5$ as the spot nears the corner at an eccentricity of $3 \mathrm{deg} 52 \mathrm{~min}$. Similar effects, but of a much smaller magnitude, were found at 0 deg $12 \mathrm{~min}$ of eccentricity.

As the spot was moved further from the corner, border effects on threshold diminished, and at $X=15$ had returned to the levels found for no border (base level). When $\mathrm{Y}=15$ at either eccentricity, base level behavior was also exhibited, indicating that the border was having an effect up to about $21.75 \mathrm{~min}$ of visual angle from the border. (Each $\mathrm{cm}$ on the screen $=1.45 \mathrm{~min}$ of visual angle.) This is equivalent to $105 \mu$ on the retina using Gullstrand's No. 2 (simplified) schematic eye with the nodal point at $16.5 \mathrm{~mm}$ (Emsley, 1936). 
Note the closely related values for points above and below the corner (i.e., $\mathrm{Y}=5$, and $Y=5 ; Y=10$ and $Y=-10)$ for eccentricity equal to $3 \mathrm{deg} 52 \mathrm{~min}$. At $\mathrm{X}=0$ the values are almost identical. This would seem to indicate that the corner and not the border is the critical factor, since the minus coordinates are not adjacent to the border. This finding is in accord with previous work (Payne \& Anderson, 1969b; Werner, 1935).

A second black figure was added to the display in Quadrant 3 [upper right-hand corner at $(-1,-1)]$ to obtain the data plotted in Fig. 3. The effect of nearing the border is clearly seen. Subjective background brightness at points away from the border returned to base level values, and, as before, the effects were visible at 0 deg $12 \mathrm{~min}$, but to a much smaller extent than at $3 \mathrm{deg} 52 \mathrm{~min}$.

Comparing the results in Fig. 3 with those in Fig 2, it is evident that the single corner gives rise to greater inhibitory effects than does the two-figure display. That is, the background was subjectively brighter at the point $(0,0)$, for example, with a display of one border (rectangle) than it was with a display of two borders (rectangles). This is consistent with the findings of Fry and Bartley (1935) on the blocking of interaction between a test spot and an inducing border by another border in the field. A similar decrease in subjective background brightness when a second border was introduced was found by Payne and Anderson (1969a). This finding adds weight to the conclusion reached above as to the importance of a corner in producing changes in subjective background brightness.

A third black figure was added to Quadrant 4 by placing its upper left hand corner at $(1,-1)$. (See insert in Fig. 4.) As in the previous figures, note differences in subjective background brightness as the border is approached, larger differences as the corner is neared, and larger differences at a retinal location further removed from the fovea.

\section{Part Two}

The second part of the experiment involved the determination of changes in subjective background brightness in a display consisting of a single rectangular figure at a large number of different retinal locations. Figure 5 presents such results for seven retinal locations. The data are for a single $Y$ coordinate, $Y=0$, with $X$ ranging from 14 to -14 in $2 \mathrm{~cm}$ steps. The range of retinal locations was $0 \mathrm{deg} 12 \mathrm{~min}$ to $3 \mathrm{deg} 52 \mathrm{~min}$ at the parafoveal region of the upper temporal retina.

Several things are evident from looking at the results in Fig. 5. First, there is a

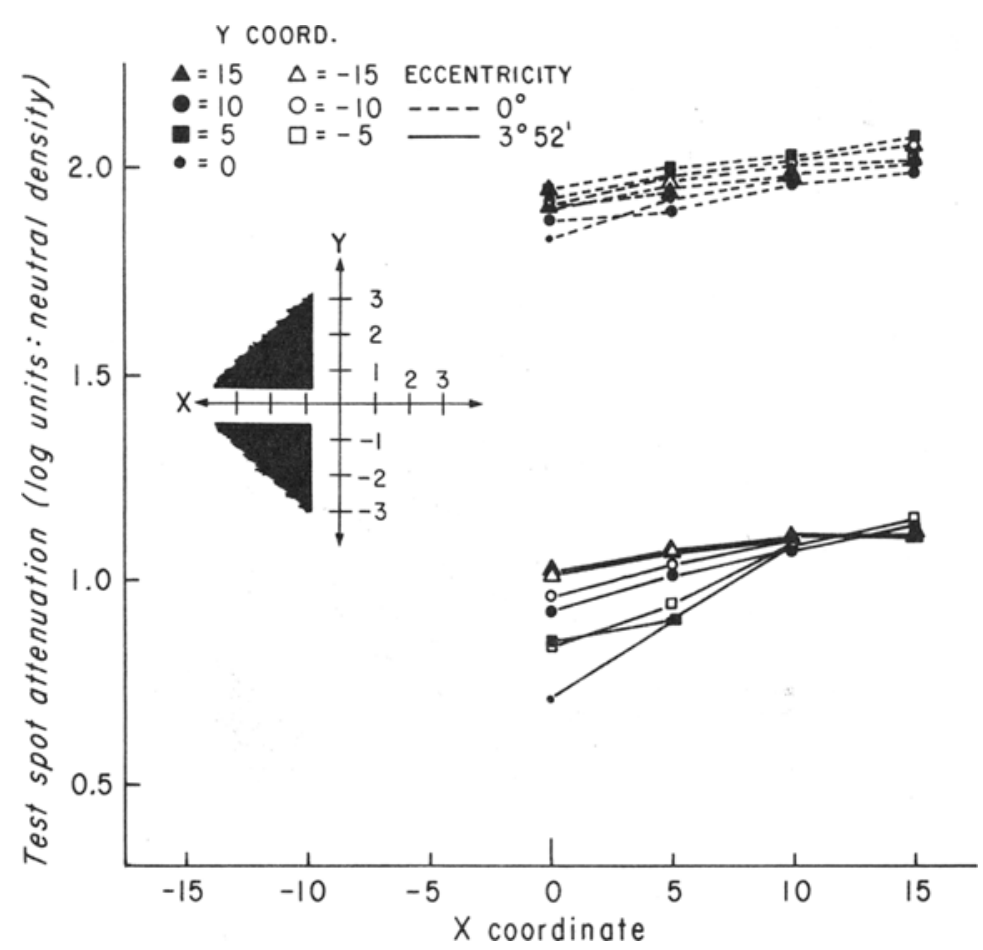

Fig. 3. Amount of attenuation (measured in desnity units) of the light source necessary to produce a brightness match between the test spot and the background as a function of the location of the test spot. Display consisted of two borders (rectangles).

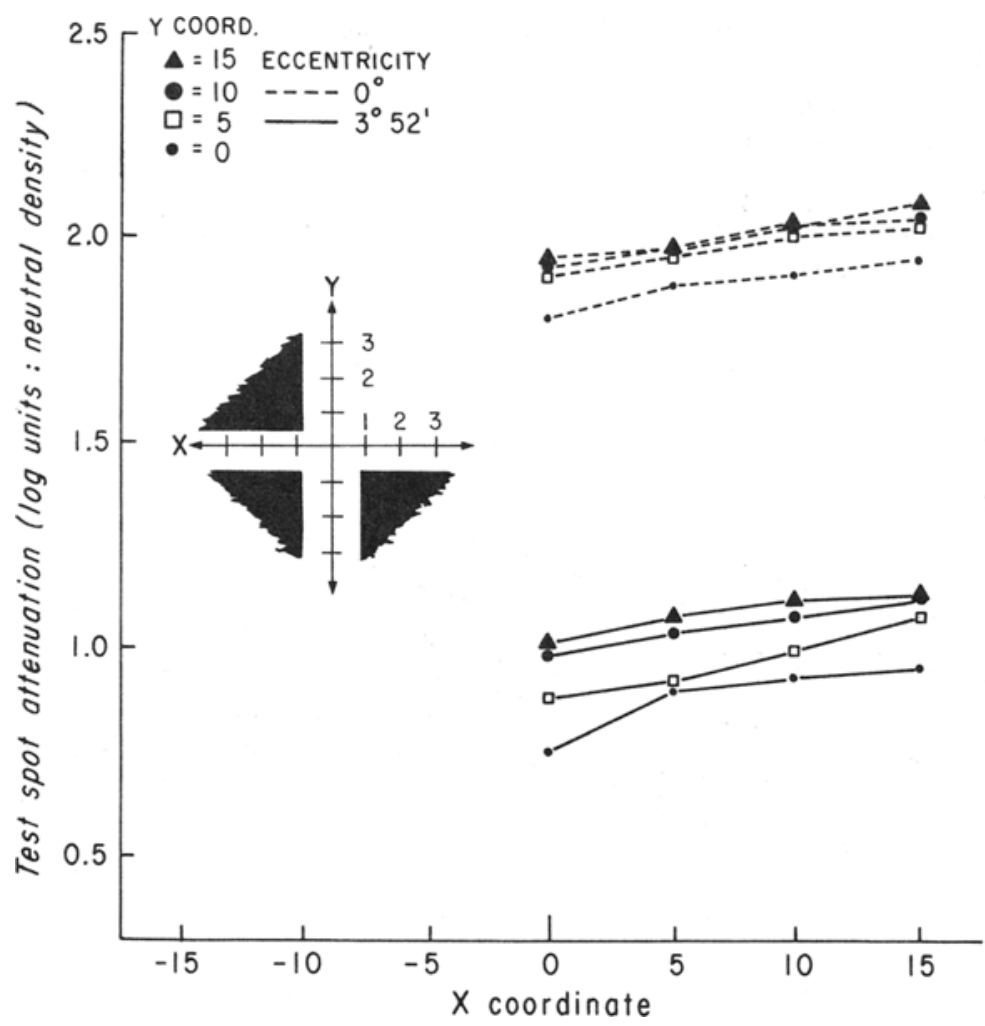

Fig. 4. Amount of attenuation (measured in density units) of the light source necessary to produce a brightness match between the test spot and the background as a function of the location of the test spot. Display consisted of three borders (rectangles). 


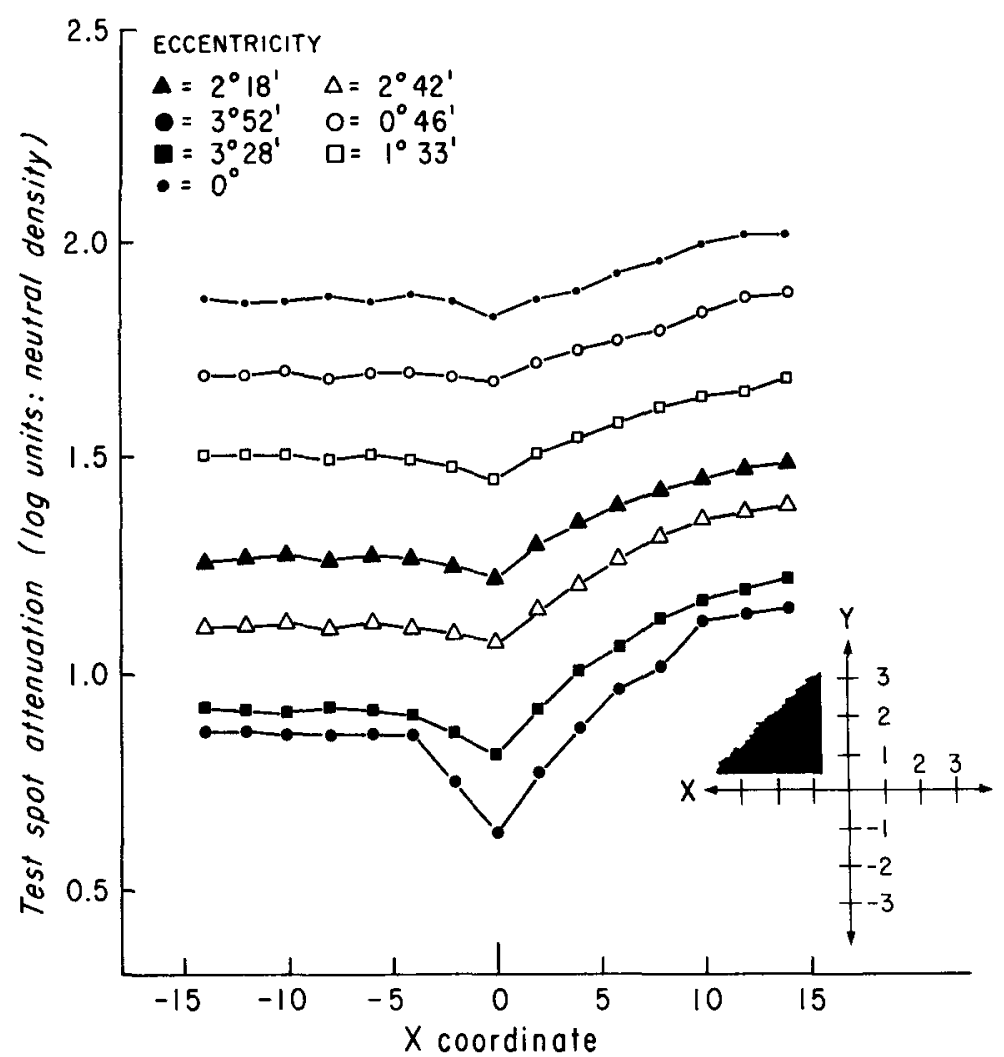

Fig. 5. Amount of attenuation (measured in density units) of the light source necessary to produce a brightness match between the test spot and the background as a function of the location of the test spot. $\mathrm{Y}=\mathbf{0}$ at all points. Display consisted of one border (rectangle).

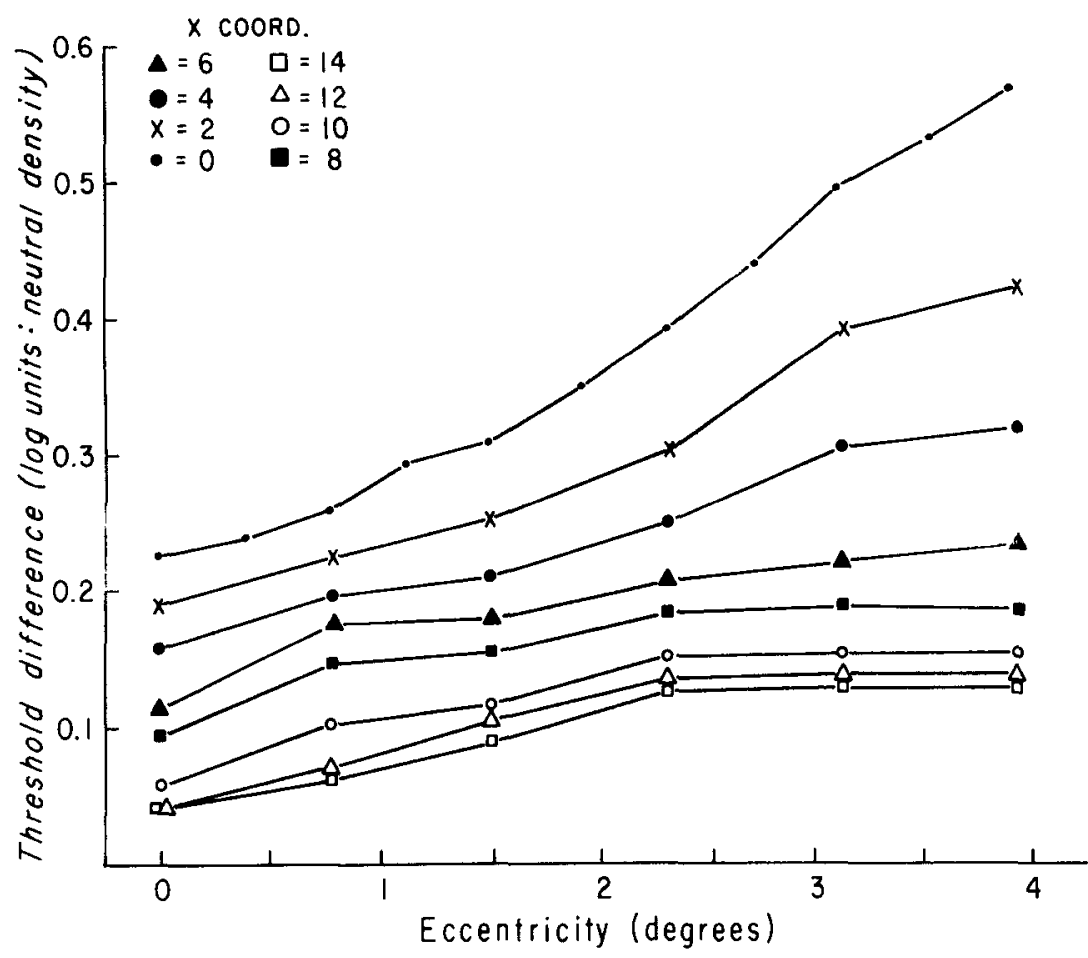

Fig. 6. Difference between base level (no border) and display (one border) as a function of retinal locus for eight $X$ coordinates. $Y=0$ at all points. progressive increase in subjective background brightness as the test spot is moved nearer the corner $(X=0)$. Second, this brightening becomes more pronounced the further the display is moved from the fovea. (In Fig. 5 this involves moving from the top of the graph to the bottom.) This point is still more clearly seen in Fig. 6.

To obtain the results presented in Fig. 6 , the following computations were performed: Base level subjective background brightness (no border in the field) was determined at the same coordinates as those given in Fig. 5 for each retinal location indicated. This base level value was then subtracted from the density value given for that same point in Fig. 5 and the results plotted as Fig. 6 .

\section{DISCUSSION}

Remembering that the base level values represent subjective background brightness due to changes in sensitivity at different retinal locations and are not due to any effect of a border, the subtraction of base level values results in curves which reflect effects due to border only.

In Fig. 6 it is obvious that the border effect becomes much greater with movement of the display away from the fovea, especially when the spot is close to the corner, e.g., $X=0, X=5$. In fact, after examining the changes in slope of the curves in Fig. 6 , it can be said that the rate of change of subjective background brightness from baseline to maximum increases with increased distance from the fovea.

\section{REFERENCES}

ALPERN, M. Metacontrast. Journal of the Optical Society of America, 1953, 43. 648-657.

AULHORN, E., \& HARMS, H. Untersuchurgen ueber das Wesen des Grenzkontrastes, Bericht ueber die 60 Zusammen kunft der Deutschen Ophthalmologischen Gesellschaft. Heidelberg, $1956,7-10$.

BLACHOWSKI, S. Studien ueber den Binnenkontrast. Zeitschrift für Psychologische und Physiologische Sinnesorganes, 1913, 2 Abt. $47,291-330$.

BROWN, J. L., \& MUELLER, C. G. Brightness discrimination and brightness contrast. In $\mathrm{C}$. H. Graham (Ed.), Vision and visual research. New York: Wiley, 1965. Pp. 208-250.

EMSLEY, H. Visual optics. Liverpool: Tinling, 1936.

FIORENTINI, A., JEANNE, M., \& TORALDO DI FRANCIA, G. Mesures photometriques visuelles sur un champ à gradient d'éclairement variable. Optica Acta, 1955, 1, 192-193.

FRY, G. A., \& BARTLEY, S. H. The effect of one border in the visual field upon the threshold of another. American Journal of Physiology, 1935, 112, 414-421.

HARMS, H., \& AULHORN, E. Studien ueber den Grenskontrast. I. Mitteilung, Ein neues Grensphanomen. Graefes Archiv für Ophthalmologie, 1955, 157, 3-23.

MACH, E. Ueber die Wirkung der raumlichen Vertheilung des Lichtreizes auf die Netzhaut, 
I. Sitsungsber. Akad. Wissenschaft, Wien, Math-nat. K1., 1865, 1895, 52, II, 303-322. PAYNE, W. H., \& ANDERSON, D. E.
Border-visibility of the Hermann grid. Journal of the Optical Society of America, 1969a, 59, 229-231.

PAYNE, W. H., \& ANDERSON, D. E. Border visibility: Corner brightening effects on the retina. Vision Research, 1969b, 9, 1309-1313.

RATOOSH, P., \& GRAHAM, C. H. Areal effects in foveal brightness discrimination. Journal of Experimental Psychology, 1951, 42, 367-375.

STEINHARDT, $\mathrm{J}$. Intensity discrimination in the

human eye. I. Journal of General Physiology, (Accepted for publication May 28, 1970).
1936, 20, 185-209.

WERNER, H. Studies on contour: I. Qualitative analysis. American Joumal of Psychology, $1935,47,40-64$. 